\title{
Investigation of the Physical and Mechanical Properties of ABS Plastic for the Additive FDM Process in the Manufacture of Models for Experiments in Wind Tunnels
}

\author{
Alexander Volkhonsky ${ }^{1, a^{*}}$, Kirill Dudkov ${ }^{1, b}$ and Reza Norollahisomarin ${ }^{1, c}$ \\ ${ }^{1}$ Moscow Aviation Institute (National Research University), Moscow, Russian Federation \\ avolkhonsk@mail.ru, bjdg4917@gmail.com, cNorollahi_reza@mail.ru
}

Keywords: Additive Technologies, FDM Layer-By-Layer Synthesis Technologies, LayerBy-Layer Overlay of Molten Polymer Filament, 3D Printer, ABS Plastic, Physical and Mechanical Properties, Sample, Tests, Temperature, Fracture, Models for Experiments in Wind Tunnels

\begin{abstract}
Work has been carried out to determine the physical and mechanical properties of ABS plastics for the manufacture of aerodynamic models using the additive technology of layer-bylayer synthesis FDM for testing in wind tunnels. The samples were tested at normal and negative temperatures. The features of the structure of the samples and the features of their destruction are revealed. The possibility of using ABS plastic for the manufacture of various models used in experiments in wind tunnels is evaluated.
\end{abstract}

\section{Problem statement}

The analysis of aircraft and rocket and space technology products shows that modern aircraft, and in particular objects for testing in wind tunnels, are characterized by a variety of materials used, small series of manufacture, consist of parts with a wide range, complex geometric shape and use a wide range of technologies that carry a huge fleet of various equipment. The FDM printer, which performs 3D printing by layering molten polymer filament (Fused Deposition Modeling - FDM), allows for the manufacture of these models to print plastic parts of a given configuration, taking into account their design features (holes, grooves, etc.), increase the accuracy of the dimensions of the working surface, expand functionality, increase reliability, resource, and improve other performance characteristics of the product as a whole, not to mention reducing production time $[1,2]$.

One of the most popular thermoplastic copolymers in the production of complex molded products requiring high manufacturing accuracy is ABS plastic (acrylonitrile butadiene styrene) thermoplastic triple copolymer of styrene, acrylonitrile and butadiene, a resistant and durable polymer suitable for the manufacture of parts for various purposes using FDM technology and is the best material for working with a 3D printer [3].

\section{Introduction}

The material of the models obtained by FDM technology consists of a set of volumes with unidirectional stacked threads. Therefore, to determine the strength properties of the material, it is necessary to investigate the strength of a model material with unidirectionally laid threads and to assess the anisotropy of the properties, tests should be carried out along and across the direction of laying the threads. The physical and mechanical characteristics of the selected ABS plastics for samples are given in Table 1.

The samples were printed on a MakerBot Replicator 2x Experimental 3D printer with a print area of $24.6 \times 15.2 \times 15.5 \mathrm{~cm}$, a layer resolution of 100 microns and an extruder nozzle diameter of 
$0.4 \mathrm{~mm}$ [6]. REC ABS plastic samples for tensile testing (at normal, elevated and lowered temperatures) they were manufactured according to GOST 11262-2017 "Plastics. Tensile test method" (Figure 1). For impact strength tests, ten samples were made in accordance with GOST 4647-2015 "Plastics. The method of determining the impact strength by Sharpie". A sample of type B (with an incision) is selected, the parameters of the samples are shown in Figure 2.

Table 1. Physical and mechanical characteristics of REC ABS plastic in comparison with analogues

\begin{tabular}{|l|c|c|c|}
\hline \multicolumn{1}{|c|}{ Indicator Name } & ABS-plastic REC & ABS-M30 & Injection Molded ABS \\
\hline Density [g/cm3] & $1.03^{*}$ & 1.04 & 1.04 \\
\hline Tensile strength [MPa] & $33 \pm 7$ & 36 & $35-50$ \\
\hline Elongation [\%] & 1,8 & - & 4 \\
\hline Young's modulus [MPa] & $1168 \pm 515$ & 2413 & $1700-2930$ \\
\hline
\end{tabular}

* This density was obtained using the hydrostatic weighing method and is not a valid value due to the presence of pores/voids in the samples.

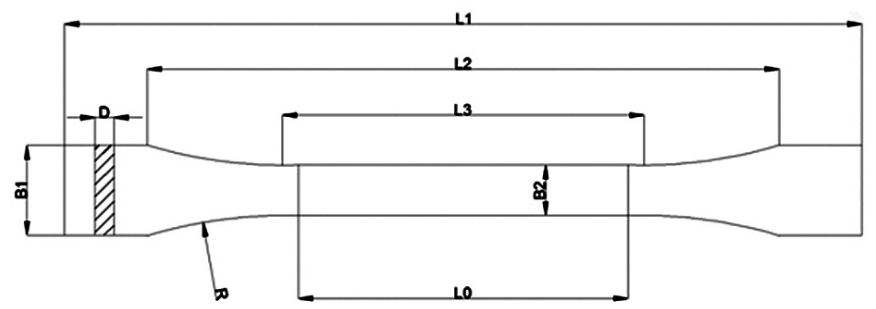

Fig. 1. Sketch of a sample for conducting tensile tests

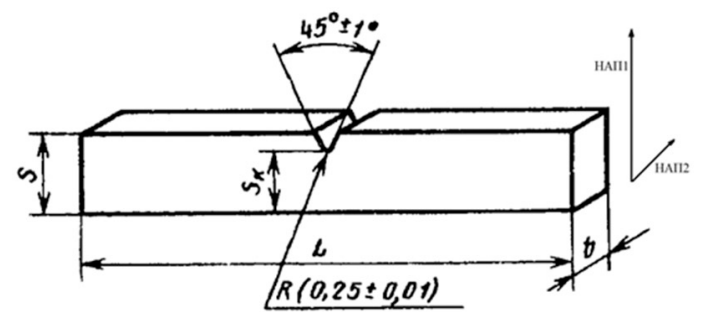

Fig. 2. Sketch and parameters of the impact strength test sample

\section{Tensile testing of ABS plastic samples at normal, elevated and lowered temperatures}

The input control of the samples was carried out using an electronic caliper MATRIX with a measurement accuracy of $\pm 0.01 \mathrm{~mm}$ (GOST 166-1989).

The tests were carried out in accordance with the methods of GOST 11262-2017 "Plastics. Tensile test method" and GOST 9550-81 "Plastics. Methods for determining the modulus of elasticity in tension, compression and bending". Tests at normal temperature were carried out on the IR breaking machine-5047-50-11. Tests at elevated and lowered temperatures were carried out on a bursting machine IR-5047-50-11. To ensure the set temperature in the test area of the samples, a thermal cabinet STI-1 was connected to the bursting machine.

Three samples of REC ABS plastic were subjected to tensile tests at normal temperatures from $+22{ }^{\circ} \mathrm{C}$ to $+27{ }^{\circ} \mathrm{C}$. The tensile strength was $33 \pm 7 \mathrm{MPa}$, the tensile strength was $32 \pm 6 \mathrm{MPa}$, the modulus of elasticity was $1168 \pm 515 \mathrm{MPa}$. 
Tensile tests at elevated temperatures from $+70{ }^{\circ} \mathrm{C}$ to $+80{ }^{\circ} \mathrm{C}$ (the temperature was selected based on the literature data $[4,5]$ ) with an exposure time of 15 minutes were subjected to samples from REC ABS plastic in the amount of 3 pieces.

The tensile strength was $21 \pm 3 \mathrm{MPa}$, the tensile strength was $17 \pm 8 \mathrm{MPa}$.

Tensile tests at a negative temperature of $-60{ }^{\circ} \mathrm{C}$ in nitrogen vapor with an exposure time of 15 minutes (the mode is selected based on literature data) samples from REC ABS plastic were subjected in the amount of $4 \mathrm{x}$.

The tensile strength was $41 \pm 18 \mathrm{MPa}$, the modulus of elasticity was $1550 \pm 308 \mathrm{MPa}$.

When trying to test the sample at a temperature of $-196^{\circ} \mathrm{C}$ in a snap-in that ensures a constant stay in liquid nitrogen with an exposure of 15 minutes, the sample collapsed during its installation in a bursting machine without fixing the moment of destruction. An optical study of the destruction sites was carried out on the destroyed sample.

\section{Testing of samples made of ABS plastic for impact strength}

The MK-30 pendulum coper was used for testing. In accordance with the GOST 4647-2015 methodology, when the sample is destroyed, the minimum reduction in impact energy should be at least $10 \%$. To do this, the impact mechanism of the pendulum copra was installed at different impact heights to perform the technique. Due to the design features of the MK-30 pendulum copra, the reduction in impact energy, even from the lowest possible height, was less than $10 \%$.

It was not possible to obtain reliable values of impact strength. To obtain reliable data, it is necessary to test samples on pendulum copra with lower impact energy and higher sensitivity.

\section{Identification of structural features of ABS plastic samples}

The visual inspection of the samples made of ABS plastic from REC company that passed the tests was carried out using optical devices (a camera and an Olympus SZX10 digital microscope).

During the visual inspection, it was revealed that the surface of the samples is white with a gloss that makes visual inspection difficult, while:

- the bottom layer, which was applied first to the technological platform of the 3D printer, is smooth, repeats its surface. If dirt particles or irregularities were present on the surface of the platform, then depressions and defects are formed on the lower layer, repeating the irregularities;

- the upper layer has a ribbing, the lateral surface of the samples also has this defect (Figure 3). The number of layers on the side surface of the sample corresponds to twenty, which corresponds to the application of the material in layers $0.2 \mathrm{~mm}$ thick.

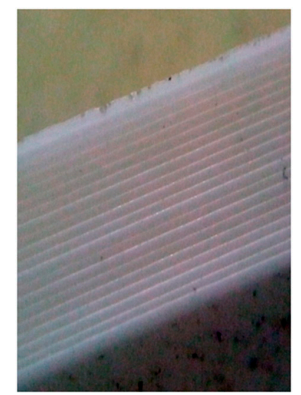

a)

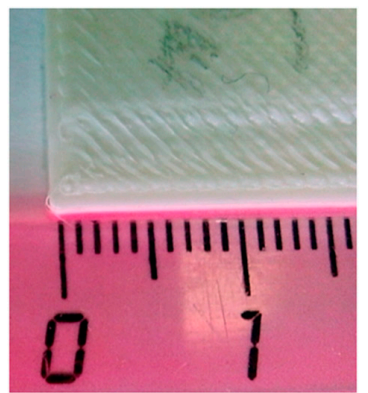

b)

Fig. 3. Photos of the ribbing on the side and upper surfaces of the sample a) - the lateral surface of the sample with a thickness of $4 \mathrm{~mm} ; \mathrm{b}$ ) - swellings on the upper surface 
In order to determine the interlayer contact, some samples were destroyed by striking with a tool that creates delaminating forces in two directions: perpendicular and parallel to the plane of the layers. The material withstands a blow perpendicular to the plane of layer construction with deformation, a blow parallel to the plane of layer construction led to the destruction of the sample into two parts (Figure 4). The interlayer boundary and the ordered placement of internal structures are clearly visible, which makes it possible to declare the non-monolithicity of the samples and the presence of oriented pores in the sample structure.

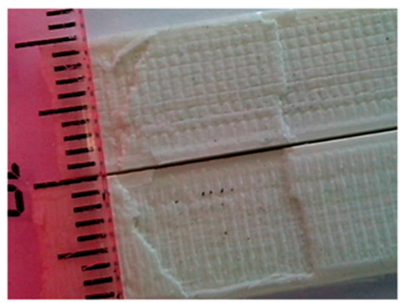

a)

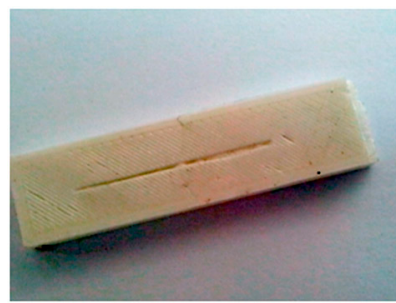

b)

Fig. 4. Photos of parts of samples after impact with a tool that creates delaminating forces in two directions: $a$-impact perpendicular to the layers; b) -impact parallel to the layers

Figure 5 shows the place of destruction of the sample during a tensile test at a temperature of $196{ }^{\circ} \mathrm{C}$ and an exposure time of 15 minutes. At very low temperatures, there are no plastic deformations in polymer materials during destruction, and they break down brittle. Accordingly, the internal structure of the sample is visible in the places of destruction without plastic deformations. Triangular-shaped pores with a base from 60 to 90 microns are clearly visible. These pores are formed during the creation of the layer and are regular throughout the entire volume of the sample. A single applied structural element (a thread with a D-shaped profile) is clearly fixed. The lower platform of the structural element occupies approximately 320 microns, the upper platform is $\approx 380$ microns, the lateral contact between the structural elements is up to a pore of160160 microns with a layer thickness of $\approx 200$ microns.

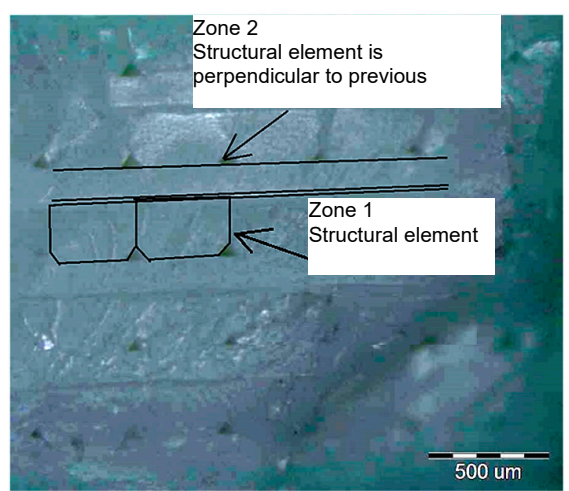

Fig. 5. Photo of the place of destruction of the sample after mechanical action during installation in the bursting machine at a temperature of $-196{ }^{\circ} \mathrm{C}$

In addition, the orientation of individual elements is clearly fixed. You can easily allocate 2 zones:

- zone 1 - a chain of threads (shaped like the letter D), the layer has a direction to the observer; 
- zone 2 is a strip with a light (side surface of a single element) and a dark (pore area) area, the layer has a direction along the drawing.

The hydrostatic weighing method determined the density $1.03 \mathrm{~g} / \mathrm{cm}^{3}$, however, due to the presence of air pores in the material, it is likely that the obtained value is not correct. The pores pass through the entire material in directions that coincide with the orientation of the structural elements, so the declared technological mode of 3D printing "100\% filling" actually corresponds to approximately $\approx 95 \%$. It was not possible to obtain photos of the destruction site at the edge of the sample due to the glossy surface of the material and large height differences (focusing optical devices does not give a clear image).

Figure 6 shows photos of the destruction of REC ABS plastic samples at normal and elevated temperatures. The internal structure is clearly visible, which (in comparison with Figure 5) is distorted by deformation from the mechanical load of the sample during testing.

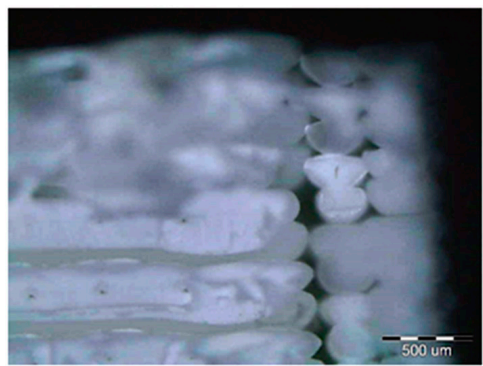

a)

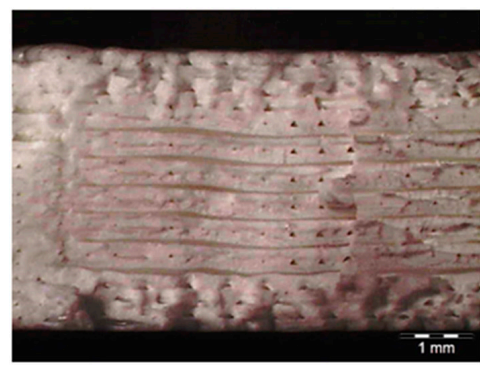

b)

Fig. 6. Places of destruction of samples during tensile tests at positive temperature: a) - photo of the place of destruction near the lateral edge, the separation of structural elements after stretching is visible; b) - the place of destruction, capturing all layers, layers with different orientations are visible

Analyzing Figure 6, it is possible to determine the distribution and orientation of structural elements along the cross-section of the sample (Figure 7).

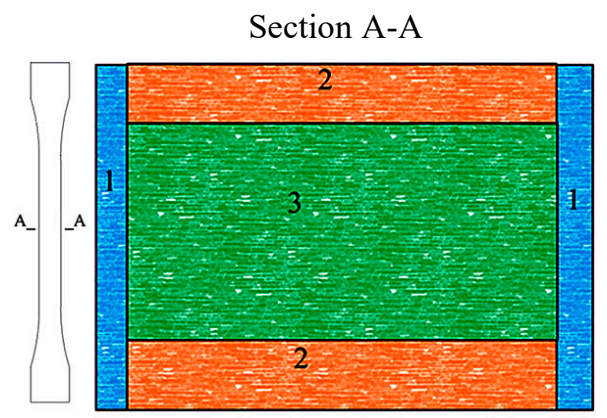

Fig. 7. Distribution and orientation of structural elements in the sample (the sample has 20 layers with a thickness close to $0.2 \mathrm{~mm}$ ):

Zone 1. Structural elements forming the contour of the layer during the construction of the sample.

Zone 2. Outer surface, thickness is 4 layers with alternating orientation of layers of structural elements $\pm 45^{\circ}$.

Zone 3. The internal structure of the sample, the thickness is 12 layers with alternating orientation of layers of structural elements $0^{\circ}$ and $90^{\circ}$ 


\section{Results and discussion}

Based on the tests of ABS plastic, the following conclusions can be drawn. Using 3D printing, it is possible to obtain plastic parts of a given configuration, taking into account structural features, in relation to the manufacture of models for testing in wind tunnels with different flow rates. The mechanical characteristics and features of the internal structure of the ABS plastic material of various brands are determined. During the tensile testing of the samples at normal temperature, an average tensile strength of $30 \mathrm{MPa}$ was obtained, which is approximately $70 \%$ of the strength of the injection grades (the average tensile strength of injection grades is $42.5 \mathrm{MPa}$ ) and $83 \%$ of the Polish equivalent of the ABS-M30. The average value of the tensile modulus of elasticity is 1570 $\mathrm{MPa}$, which is approximately $70 \%$ of the modulus of elasticity of injection molding grades (the average value of the modulus of elasticity of injection molding grades is $2300 \mathrm{MPa}$ ) and $65 \%$ of the Polish equivalent of the ABS-M30. The lag of ABS plastic from injection-molded ABS brands is due to a feature of 3D printing technology, namely, layer-by-layer application of the material.

In the process of tensile testing of samples at elevated temperatures, an average tensile strength of $21 \mathrm{MPa}$ was obtained (it drops by $30 \%$ compared to the tensile strength at normal temperature).

When testing the samples for tensile strength at a reduced temperature, an average tensile strength of $41 \mathrm{MPa}$ was obtained (it increases by $37 \%$ compared to the tensile strength at normal temperature) and the average tensile modulus of $1550 \mathrm{MPa}$ (compared to the elastic modulus at normal temperature practically does not change).

The temperature range of the tests (from $-60^{\circ} \mathrm{C}$ to $+70^{\circ} \mathrm{C}$ ) was wider than the operating interval from $-40^{\circ} \mathrm{C}$ to $+75^{\circ} \mathrm{C}$ and shows that when the temperature drops to $-60{ }^{\circ} \mathrm{C}$ the material is operable. The peculiarity of this material is the presence of several links in the molecular chain of the material (styrene, butadiene and acrylonitrile), which complicates the construction of a complete picture of the behavior of the material. To clarify the temperature of frost resistance, a number of different tests are required at lower temperatures.

In the process of optical examination of the destruction sites, the following structural features of the samples were revealed:

The temperature range of the tests (from $-60^{\circ} \mathrm{C}$ to $+70^{\circ} \mathrm{C}$ ) was wider than the operating interval from $-40{ }^{\circ} \mathrm{C}$ to $+75^{\circ} \mathrm{C}$ and shows that when the temperature drops to $-60{ }^{\circ} \mathrm{C}$, the material is operable. The peculiarity of this material is the presence of several links in the molecular chain of the material (styrene, butadiene and acrylonitrile), which complicates the construction of a complete picture of the behavior of the material. To clarify the temperature of frost resistance, a number of different tests are required at lower temperatures.

In the process of optical examination of the destruction sites, the following structural features of the samples were revealed:

- during the application of the layer, pores are formed along the thread of the material throughout the part (the presence of pores raises the question of water entering the pores and turning it into ice at negative temperatures with the creation of tension from the inside);

- the pores have a shape close to triangular, with the side of the base of the triangle from 60 to 90 microns and a height of 40 microns;

- filling of samples with $100 \%$ 3D printing settings is actually approaching $95 \%$ due to the peculiarity of the technology - the pores formed;

- anisotropy of the strength index is observed depending on the direction. Interlayer strength is the weakest part of this material, which must be taken into account when manufacturing products using 3D printing technology. 


\section{Conclusions}

The conducted studies have shown the possibility of using additive technologies and ABS plastic for the manufacture of various geometrically similar models of aircraft and rocket and space technology for testing in wind tunnels. The use of such models significantly reduces the actual cycle of their manufacture - from one year to several days, reduces manufacturing costs by more than $60 \%$, reduces the volume of documentation for the manufactured model by an order of magnitude, and also increases the reliability of their design. However, due to the presence of pores in the structure of ABS plastic, which is a feature of FDM technology, it is impossible to manufacture drained aerodynamic models, since the material does not ensure their tightness. Samples made of ABS plastic have a rough surface and require additional processing to ensure a given purity. To create models with high requirements, it is necessary to use a different type of technology, or the use of a material that ensures the absence of internal defects and high quality geometry.

\section{References}

[1] Volkhonsky, A.E., Gudkov, K.V. Methods of manufacturing prototypes and parts of aggregates of various industrial products using additive technologies. // Educational Technologies, No.1, 2014, pp. 127-143.

[2] Panferova, E.A., Strukov, A.N., Strukova, A.V., Mishchenko, V.Yu. Methods of prototyping products in the aerospace industry and the equipment used. // Flight, 2013, No. 7. pp. 29-33.

[3] Volkhonsky, A.E., Dudkov, K.V. Manufacturing of "pad" type parts by FDM prototyping. // Polymer Materials, No.3 (178), 2014. pp. 42-45.

[4] Astapov, V.Yu., Khoroshko, L.L., Giordani, M.S., Khoroshko, A.L. CAD in modeling the modes of technological processes for the production of structural elements of aircraft // Proceedings of MAI. 2016. No. 87. URL: http://trudymai.ru/published.php?ID=69638

[5] Laboratory of three-dimensional printing. [Electronic document] - Access mode: http://www.lab3dprint.ru/support/425-abs-vs-pla.

[6] MakerBot Replicator+3D printer. [Electronic document] - Access mode: https:/www.makerbot-gr/shop-makerbot-products/makerbot-replivator-2x-experimental-3dprinter/ 